\title{
CROISSANCE PROLONGÉE DE LA DIATOMÉE MARINE PHAEODACTYLUM TRICORNUTUM EN MILIEU CONTAMINÉ PAR LE CHLORURE DE TRIBUTYLÉTAIN : CONSÉQUENCE SUR LE BIOTRANSFERT DU POLLUANT.
}

\author{
P. MARSOT* (1), K. MOUHRI (2), E. PELLETIER (1), N. SAINT-HILAIRE (3), \\ M. LOUDIKI (2), J. PELLERIN (3).
}

(1) Institut National de la Recherche Scientifique (INRS-Océanologie), Rimouski G5L 3A1, Québec, Canada.

(2) Département de Biologie, Faculté des Sciences Semlalia, B.P. S 15, Marrakech, Maroc.

(3) Département d'Océanographie, Université du Québec à Rimouski, Québec, Canada.

\section{RÉSUMÉ}

Cette étude consiste à évaluer le biotransfert du tributylétain (TBT) par le filtreur bivalve Mya arenaria, à partir d'une culture semi-continue de la diatomée Phaeodactylum tricornutum exposée à un niveau de contamination n'arrêtant pas sa croissance à long terme. La dose du contaminant employée (100 $\left.\mathrm{ng}^{-1}\right)$ est de l'ordre de grandeur de celles décelées dans certaines régions contaminées, telles les marinas et les régions portuaires. Dans ces conditions, la culture de $P$. tricornutum a maintenu son équilibre de croissance durant plus de 50 jours. Sa productivité était légèrement inférieure (de $4 \%$ ) à celle de la culture témoin (sans contaminant). L'ingestion des diatomées contaminées, sur une période de 90 jours, a entraîné une bioaccumulation de TBT et DBT (dibutylétain) dans la chair du mollusque, atteignant des concentrations respectives de 133 et $128 \mathrm{ng} \mathrm{g}^{-1}$ (poids humide). L'étude suggère que le biotransfert du TBT et du DBT associés à des micro-algues en croissance continue ou semi-continue pourrait constituer un facteur de bioamplification important du biocide en milieu naturel.

Mots-clés : tributylétain, diatomée marine, Phaeodactylum tricornutum, biotransfert, mollusques bivalves.

\section{LONG-TERM GROWTH OF THE MARINE PHAEODACTYLUM TRICORNUTUM EXPOSED TO TRIBUTYLTIN CHLORIDE : CONSEQUENCE ON BIOTRANSFER OF THE POLLUTANT.}

\begin{abstract}
The aim of this study was to examine the uptake and the accumulation of tributyltin (TBT) by the filter-feeding bivalve Mya arenaria, from a semi-continuous culture of Phaeodactylum tricomutum contaminated with TBT at a concentration which did not stop its long-term growth. By using contamination level commonly found in natural polluted areas (100 ng TBT 1-1), a long term growth was achieved by the algal culture, with a productivity reduced by only $4 \%$. The accumulation
\end{abstract}

\footnotetext{
* Auteur à qui la correspondance doit être adressée.
} 
of TBT and DBT (dibutyltin) by the bivalve occurred during the ingestion of microalgae and increased continuously during the 90-days exposure period. The total body concentrations of TBT and DBT compounds reached, at the end of the experiment, 133 and $128 \mathrm{ng} \mathrm{g}^{-1}$ (wet mass) respectively. The biotransfer of organotins associated with continuous growing microalgae in an open system (continuous or semi-continuous culture) is an important factor to be considered in the risk assessment of the biocide in the natural environment.

Key-words : tributyltin, marine diatom, Phaeodactylum tricornutum, biotransfer, bivalve molluscs.

\section{INTRODUCTION}

Les composés organiques de l'étain tels les trialkyles (tributylétain et triphénylétain) sont utilisés comme agents biocides dans les peintures anti-salissures de bateaux, d'engins de pêche et dans la pratique agricole (EVANS et SMITH, 1975). Malgré les restrictions nationales limitant leur emploi pour les bateaux de moins de $25 \mathrm{~m}$, les niveaux de contamination retrouvés dans les zones portuaires atteignent parfois de 100 à $800 \mathrm{ng} \mathrm{l}^{-1}$ (ALZIEU et al., 1991). L'étude des effets nocifs des organoétains sur le phytoplancton mérite une attention particulière puisque ces microorganismes constituent le premier maillon de la chaine trophique et leur ingestion par les organismes consommateurs peut occasionner une bioamplification du polluant dans le milieu aquatique (LAUGHLIN et al., 1986). L'effet nocif de ces composés sur la reproduction des microalgues se révèle, en culture confinée, à des concentrations variant entre 100 et $500 \mathrm{ng} \mathrm{l}^{-1}$ (WONG et al., 1982 ; WALSH et al., 1985 ; LIYING et al., 1990 ; HUANG et al., 1993). Les diatomées marines sont particulièrement sensibles aux biocides. Ainsi, la croissance de Skeletonema costatum est affectée par des concentrations aussi faibles que $100 \mathrm{ng} \mathrm{l}^{-1}$ (BEAUMONT et NEWMAN, 1986). Les études récentes de SAINT-LOUIS et al. (1994) et MARSOT et al. (1995) démontrent que les micro-algues sont plus tolérantes envers ce produit en milieu ouvert (culture continue). De plus, les micro-algues peuvent maintenir une croissance en état d'équilibre dans un milieu modérément contaminé $\left(<8 \mu \mathrm{g} \mathrm{I}^{-1}\right)$ par le tributyl ou le triphénylétain. Dans la mesure où la culture continue reflète davantage le milieu naturel (RHEE, 1980), la croissance persistante de micro-algues contaminées constitue un fait nouveau qui évoque la possibilité d'un biotransfert important du polluant à travers la chaîne alimentaire marine. Ceci vise plus particulièrement les organismes filtreurs planctoniques qui sélectionnent le matériel particulaire vivant (POULET et MARSOT, 1978 ; NEWELL et JORDAN, 1983), et les espèces benthiques qui concentrent plusieurs milliers de fois l'organoétain sous sa forme toxique (WALDOCK et THAIN, 1983 ; ZUOLIAN et JENSEN, 1989 ; HIGASHIYAMA et al., 1991).

Les études de biotransfert en laboratoire sur des mollusques bivalves montrent effectivement que la phase particulaire (micro-algues) constitue une voie de contamination plus importante que la phase aqueuse (LAUGHLIN et al., 1986). Ces essais, qui comportent l'emploi de micro-algues en croissance discontinue (culture confinée) ou exposées sur un court laps de temps à de fortes doses d'organoétains, s'éloignent toutefois de la réalité. Le présent travail vise à démontrer le transfert et la bioaccumulation du tributylétain chez un mollusque à partir de diatomées en croissance continue soumises à un faible niveau de contamination. De plus, notre étude cherche à répondre à deux questions : premièrement, un niveau de contamination au TBT, de l'ordre de celui observé in situ dans les zones contaminées, peut-il affecter la croissance continue d'une diatomée planctonique ? Deuxièmement, est-ce que cette production de diatomées contaminées peut entraîner un biotransfert et une bioaccumulation perceptibles du contaminant chez un consommateur herbivore?

\section{MATÉRIEL ET MÉTHODES}

Le mollusque Mya arenaria fut récolté dans une région très peu contaminée de l'estuaire du Saint-Laurent (Québec) : les lles du Bic, situées en amont, à environ $40 \mathrm{~km}$ du lieu d'expérimentation (laboratoire aquicole de la Pointe-au-Père, INRS-Océanologie). Les animaux 
ont été transférés dans des cages à tamis et enfouis sous $5 \mathrm{~cm}$ de sable. Les cages furent immergées dans un bassin d'une capacité de 3500 litres, recevant un apport constant d'eau de mer filtrée sur sable au débit de $1440 \mathrm{lj}^{-1}$. La salinité de l'eau de mer s'est maintenue en moyenne à $27 \%$ et sa température, dans le bassin, était de $12^{\circ} \mathrm{C}$. Une durée d'acclimatation des animaux de 2 semaines précédait le début de l'expérience. La diète algale était constituée de la diatomée Phaeodactylum tricornutum. L'abondance de cette diatomée dans notre région estuarienne (SINCLAIR, 1978) et la facilité de digestion de ce microorganisme par Mya arenaria (SHUMWAY et al., 1985) ont justifié le choix de cette espèce. La diatomée croissait en cultures semi-continues de $20 \mathrm{I}$, sur milieu enrichi ( $\mathrm{f} / 2$, de GUILLARD et RYTHER, 1962), au taux de dilution de $0,1 \mathrm{j}^{-1}$. Les cultures étaient exposées à une température de $16^{\circ} \mathrm{C}$ et à un éclairement de $300 \mu \mathrm{E} \mathrm{m} \mathrm{m}^{-2} \mathrm{~s}^{-1}$, assuré par des fluorescents " cool white ", sur une photopériode de 16h/24h. Le biotransfert du TBT a été observé sur des mollusques nourris durant 90 jours d'une culture semi-continue de la diatomée contaminée avec $100 \mathrm{ng}$ de TBT $\mathrm{l}^{-1}$ (milieu nourricier). La récolte $\left(2 \mathrm{l} \mathrm{j}^{-1}\right)$, représentant en moyenne $30,7 \times 10^{9}$ cellules $j^{-1}$, était administrée aux mollusques. Le volume de culture ainsi prélevé était remplacé par du milieu frais contaminé par-le TBT $\left(100 \mathrm{ng} \mathrm{l}^{-1}\right)$. Une culture témoin de $P$. tricornutum, soumise aux mêmes conditions de croissance mais sans contaminant, servait à évaluer l'impact du TBT sur la productivité des algues. De plus, sa récolte $\left(2 / \mathrm{j}^{-1}\right)$ constituait une diète algale non contaminée pour des animaux témoins. Une prolifération bactérienne dans les cultures de diatomées, survenant après 52 jours d'opération, nécessita le réensemencement de nouvelles cultures.

L'extraction des organoétains (tributyl et dibutylétain) de la chair des mollusques et leur analyse par chromatographie en phase gazeuse (CG-SM) ont été réalisées selon le protocole de ASHBY et CRAIG (1991), modifié par MERCIER et al. (1994). Chaque échaintillon comportait un homogénat de 5 individus. Les homogénats ont été réalisés à l'aide d'un homogénéisateưr Virtis et complétés par une sonification dans le solvant hexane contenant $0,1 \%$ (poids/volume) de tropolone. Le tripentyl ( $100 \mu \mathrm{l} / 1,5 \mathrm{~g}$ d'homogénat) servait de standard interne. L'hydrogénation était effectuée par l'addition de $\mathrm{NaBH}_{4}$ (4\% poids/volume). La fraction organique fut purifiée sur une cartouche de gel de silice, en utilisant l'hexane comme agent d'élution. L'échantillon fut réduit par évaporation à un volume final de $0,1 \mathrm{ml}$, avant son injection dans le chromatographe. Les analyses ont été faites en double, et la variation analytique des réplicats était de 10,5\% pour le TBT et de $11,6 \%$ pour le DBT.

\section{RÉSULTATS}

Les résultats présentés dans le tableau I montrent que la culture semi-continue de

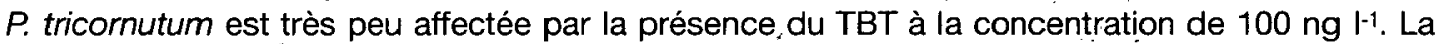
densité cellulaire de la culture contaminée est demeurée relativement constante tout au long de la période de 52 jours, à $15,4 \times 10^{9}$ cellules $\mathrm{l}^{-1} \pm 2,28$. Toutefois, un test statistique " $t$ student " révèłe une légère différence significative entre les productivités en cellules et en poids sec des cultures témoin et contaminée. Cette différence s'est avérée significative au niveau de probabilité $P<0,05$.

Tableau I : Croissance et productivité de cultures semi-continues de Phaeodactylum tricornutum, non contaminée (témoin) et contaminée au TBT (100 $\mathrm{ng} \mathrm{l}^{-1}$ ), sur une période de 52 jours. (Valeurs \pm écart type de la moyenne, $n=17$ ).

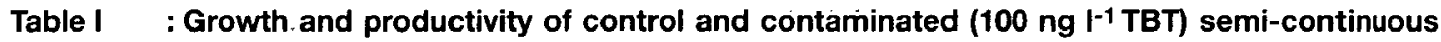
cultures of the diatom Phaeodactylum tricornutum, over a 52-days period. (Means of $17 \pm$ standard deviation).

\begin{tabular}{lcccc}
\hline Culture & $\begin{array}{c}\text { Taux de dilution } \\
\left(\mathbf{j}^{-1}\right)\end{array}$ & $\begin{array}{c}\text { Densité cellulaire } \\
\left(10^{9} \text { cell. } \mathrm{l}^{-1}\right)\end{array}$ & $\begin{array}{c}\text { Productivité } \\
\left(10^{9} \text { cell. } \mathrm{l}^{-1} . \mathrm{j}^{-1}\right)\end{array}$ & $\left(\mathrm{g} \mathrm{m} . \mathrm{s} . \mathrm{l}^{-1} . \mathrm{j}^{-1}\right)$ \\
\hline Témoin & 0,1 & $16,0 \pm 1,98$ & $1,60 \pm 0,19$ & $0,024 \pm 0,0025$
\end{tabular}

Contaminée 
L'analyse des organoétains en chromatographie montre une accumulation progressive du tributylétain et du dibutylétain dans la chair des mollusques, au cours des 90 jours de contamination par la diatomée (fig. 1). Le dibutylétain, une forme dégradée du TBT ayant perdu un groupement butyle, est présent à des concentrations parfois supérieures au TBT. La bioaccumulation est particulièrement évidente après 90 jours de traitement : les teneurs non détectables en TBT et DBT $\left(<2,5 \mathrm{ng} \mathrm{g}^{-1}\right.$ poids humide) enregistrées au temps 0 atteignent respectivement, à la fin de la période d'essai, 133 et $128 \mathrm{ng} \mathrm{g}^{-1}$.

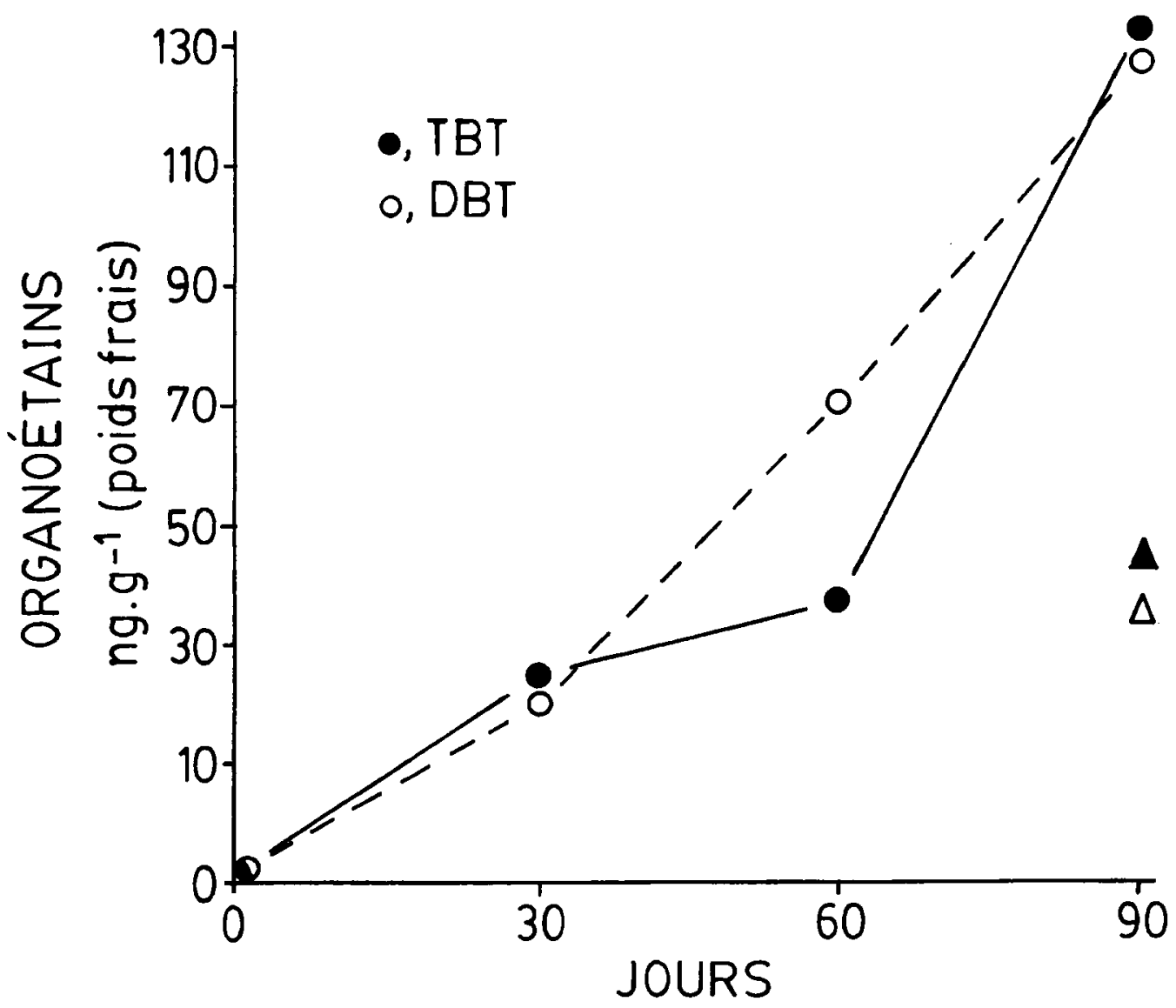

Figure 1 : Bioaccumulation du tributylétain (TBT) et du dibutylétain (DBT) par le bivalve Mya arenaria nourri, sur une période de 90 jours, de la diatomée Phaeodactylum tricornutum., 0 : animaux nourris, de diatomées contaminées avec $100 \mathrm{ng}$ de TBT $\mathrm{I}^{-1}$ (milieu nourricier de la culture semi-continue). $\Delta, \triangle$ : animaux témoins nourris de diatomées non contaminées.

Figure 1 : Accumulation of tributyltin (TBT) and dibutyltin (DBT) by the bivalve Mya arenaria fed, over a 90-days period, on the diatom Phaeodactylum tricornutum. 9,0 : animals fed with contaminated diatoms i.e. exposed to $100 \mathrm{ng} \mathrm{l}^{-1}$ TBT in semi-continuous culture. $\triangle, \triangle$ : animals fed with non-contaminated diatoms.

Par ailleurs, les mollusques témoins exposés à la même eau de mer du laboratoire mais recevant une diète algale non contaminée ont tout de même accumulé 45 et $34 \mathrm{ng} \mathrm{g}^{-1}$ (poids humide) de TBT et DBT, respectivement (fig. 1). Tenant compte du très faible degré de contamination des animaux au temps 0 , cette donnée suggère un apport non négligeable d'organoétains via le système d'amenée d'eau de mer du laboratoire. Toutefois, les analyses effectuées sur l'eau de mer et sur le milieu extracellulaire des cultures de $P$. tricornutum n'ont révélé aucune teneur détectable en organoétains $(<1 \mathrm{ng} \mid-1)$. 


\section{DISCUSSION}

L'ensemble de ces résultats démontre la capacité de reproduction à long terme des diatomées dans un milieu contaminé par le TBT et nous amène à conclure que cette activité algale pourrait constituer une voie de dispersion continue des organoétains au niveau de la chaîne trophique. La disparition du TBT du milieu extracellulaire de la culture contaminée témoigne d'une prise en charge cellulaire du contaminant par adsorption ou absorption, et détermine son association au matériel particulaire. Ce processus de décontamination de la phase aqueuse par bioaccumulation cellulaire a aussi été observé en culture continue de faible densité cellulaire, utilisant une eau de mer non enrichie (MARSOT et al., 1995) ; ce qui nous permet d'inférer les résultats aux conditions du milieu naturel. Cette " biodispersion continue » du contaminant pourrait affecter plus spécifiquement les mollusques bivalves dont les activités sélectives, basées sur des critères de taille et de qualité nutritionnelle, leur permettent de discerner une micro-algue d'une particule inerte (LOOSANOFF, 1949 ; JORGENSEN, 1975 ; HYLLEBERG et GALLUCCI, 1975). D'autres études soulignent des facteurs de bioconcentration élevés du TBT chez des moules exposées à un milieu naturel contaminé (ZUOLIAN et JENSEN, 1989), et plus particulièrement lorsque le biocide est associé au phytoplancton (LAUGHLIN et al., 1986). La propagation du TBT par les micro-algues en croissance vise aussi les filtreurs planctoniques (copépodes, larves véligères) qui, non seulement discernent les cellules vivantes lors de leur capture (POULET et MARSOT, 1978 ; LE PENNEC et RANGEL-DAVALOS, 1985), mais les repèrent à distance et s'en approchent par chimiotropotactisme (POULET et MARSOT, 1980).

La forte accumulation d'organoétains observée chez Mya arenaria (fig. 1) supporte les observations faites in situ dans les sites contaminés où l'on détecte, chez cette espèce, des concentrations plus élevées en organoétains (KURE et DEPLEDGE, 1994). $M$. arenaria peut, en effet, concentrer plus de 200000 fois le TBT dissous, probablement à cause de son inaptitude à métaboliser et à éliminer efficacement cette substance (LANGSTON et al., 1987 ; KURE et DEPLEDGE, 1994). Ces résultats suggèrent donc que les teneurs élevées de DBT, retrouvées dans la chair des mollusques (fig. 1), proviendraient des cultures de $P$. tricornutum dont la capacité de biodégradation du TBT en DBT a été démontrée récemment (MOUHRI et al., 1995). Cette propriété de dégrader le TBT en une molécule moins toxique, le DBT, existe également chez d'autres micro-algues d'eau douce (MAGUIRE et al., 1984) et marines (LEE et al., 1989 ; SAINTLOUIS et al., 1994), et contribuerait à la détoxication partielle du produit en milieu aquatique. Une partie du DBT proviendrait également de l'eau de mer du laboratoire, puisque ce composé est retrouvé chez les individus témoins. L'accumulation d'organoétains chez les individus témoins évoluant dans un milieu apparemment exempt du polluant (fig. 1), confirme le choix de Mya arenaria comme bioindicateur efficace de pollution par le TBT (KURE et DEPLEDGE, 1994). En effet, la bioconcentration d'organoétains par ce bivalve permet de révéler des teneurs de TBT à peine perceptibles dans un milieu marin.

\section{CONCLUSION}

Notre essai en culture semi-continue démontre que la croissance prolongée de diatomées dans un milieu aquatique faiblement contaminé par le TBT peut constituer une voie de dispersion continue du contaminant et, de ce fait, provoquer une bioaccumulation chez les organismes consommateurs. Cette biodispersion pourrait affecter, plus particulièrement, les filtreurs herbivores qui métabolisent difficilement le composé (ex. Mya arenaria) et/ou qui sélectionnent le matériel particulaire selon le critère de la qualité nutritionnelle.

\section{REMERCIEMENTS}

Cette recherche a été financée par l'organisme AUPELF-UREF (Association des Universités Partiellement de Langue Française) et le Fonds Institutionnel de Recherche de l'Institut National de la Recherche Scientifique. 


\section{BIBLIOGRAPHIE}

ALZIEU C., MICHEL P., TOLOSA I., BUCCI E., MEE L.D., READMAN J.W., 1991. Organotin compounds in the Mediterranean : a continuing cause for concern. Mar. environ. Res., $32,261-270$.

ASHBY J.R., CRAIG P.J., 1991. Speciation for analysis of organotin compounds by GC AA and GC MS after ethylation by sodium tetraethylborate. App. Organomet. Chem., 5, 173-181.

BEAUMONT A.R., NEWMAN P.B., 1986. Low levels of tributyltin reduce growth of marine microalgae. Mar. Pollut. Bull., 17, 457-461.

EVANS C.J., SMITH P.J., 1975. Organotin-based antifouling systems. J. Oil Colour Chem. Assoc., $58,160-168$.

GUILLARD R.R.L., RYTHER J.H., 1962. Studies of marine planktonic diatoms I. Cyclotella nana (Hustedt), and Detonula confervacea (Cleve) Gran. Can. J. Microbiol., 8, 229-239.

HIGASHIYAMA T., SHIRAISHI H., OTSUKI A., HASHIMOTO S., 1991. Concentrations of organotin compounds in blue mussels from the wharves of Tokyo bay. Mar. Pollut. Bull., $22,585-587$

HUANG C., BAI Z., DAI S., XIE Q., 1993. Accumulation and toxic effect of organometallic compounds on algae. Appl. Organometal. Chem., 7, 373-380.

HYLLEBERG J., GALLUCCI V.F., 1975. Selectivity in feeding by the deposit-feeding bivalve Macoma nasuta. Mar. Biol., 32, 167-178.

JORGENSEN C.B., 1975. On gill function in the mussel Mytilus edulis L. Ophelia, 13, 187-232.

KURE L.K., DEPLEDGE M.H., 1994. Accumulation of organotin in Littorina littorea and Mya arenaria from Danish coastal waters. Environ. Pollut., 84, 149-157.

LANGSTON W.J., BURT G.R., ZHOU M., 1987. Tin and organotin in water, sediments and benthic organisms of Poole Harbour. Mar. Poll. Bull., 18, 634-639.

LAUGHLIN R.B. Jr., FRENCH W., GUARD H.E., 1986. Accumulation of bis (tributyltin) oxide by the marine mussel Mytilus edulis. Environ. Sci. Technol., 20, 884-890.

LEE T.F., VALKINS A.O., SELIGMAN P.F., 1989. Importance of microalgae in the biodegradation of tributyltin in estuary waters. Environ. sci. Technol., 23, 1515-1518.

LE PENNEC M., RANGEL-DAVALOS C., 1985. Observations en microscopie à épifluorescence de l'ingestion et de la digestion d'algues unicellulaires chez des jeunes larves de Pecten maximus (Pectinidae, Bivalvia). Aquaculture, 47, 39-51.

LIYING Z., XIANBUN L., BINGYI S., 1990. Toxic effects of organotin on marine diatoms. J. Ocean University of Quingdao, 20, 125-131.

LOOSANOFF V.L., 1949. On the food selectivity of oysters. Science, 110, 122.

MAGUIRE R.J., WONG P.T.S., RHAMEY J.S., 1984. Accumulation and metabolism of tri-n-butyltin cation by green alga, Ankistrodesmus falcatus. Can. J. Fish. aquat. Sci., 41, 537-540.

MARSOT P., PELLETIER E., SAINT-LOUIS R., 1995. Effects of triphenyltin chloride on growth of the marine microalga Pavlova lutheri in continuous culture. Bull. Environ. Contam. Toxicol., 54, 389-395.

MERCIER A., PELLETIER E., HAMEL J.F., 1994. Metabolism and subtle toxic effects of butyltin compounds in starfish. Aquat. Toxicol., 28, 259-273.

MOUHRI K., MARSOT P., PELLETIER E., LOUDIKI M., SAINT-LOUIS R., 1995. Effets du chlorure de tributylétain sur la croissance et le métabolisme de la diatomée marine Phaeodactylum tricornutum (Bohlin). Oceanol. Acta, 18, 363-370. 
NEWELL R.I.E., JORDAN S.J., 1983. Preferential ingestion of organic material by the American oyster, Crassostrea virginica. Mar. Ecol. Prog. Ser., 13, 47-53.

POULET S.A., MARSOT P., 1978. Chemosensory grazing by marine calanoid copepods (Arthropoda crustacea). Science, 200, 1403-1405.

POULET S.A., MARSOT P., 1980. Chemosensory feeding and food-gathering by omnivorous marine copepods. In KERFOOT W.C., Evolution and ecology of zooplankton communities, 198-218, Univ. Press of New England.

RHEE G.Y., 1980. Continuous culture in phytoplankton ecology. In DROOP M.R., JANNASCH H.W., Advances in aquatic microbiology, 2, 151-203, Academic Press London.

SAINT-LOUIS R., PELLETIER E., MARSOT P., FOURNIER R., 1994. Distribution et effets du chlorure de tributylétain et de ses produits de dégradation sur la croissance de l'algue marine Pavlova lutheri en culture continue. Water Res:, 28, 2533-2544.

SHUMWAY S.E., CUCCI C.L., NEWELL R.C., YENTSCH C.M., 1985. Particule selection, ingestion, and absorption in filter-feeding bivalves. J. exp. mar. Biol. Ecol., 91, 77-92.

SINCLAIR M., 1978. Summer phytoplankton variability in the lower St.-Lawrence estuary. J. Fish. Res. Bd. Can., 35, 1171-1185.

WALDOCK M.J., THAIN J.E., 1983. Shell thickening in Crassostrea gigas : organotin antifouling or sediment induced? Mar. Pollut. Bull., 14, 411-415.

WALSH G.E., MCLAUGHLAN L.L., LORES E.M., LOUIE M.K., DEANS C.H., 1985. Effects of organotins on growth and survival of two marine diatoms, Skeletonema costatum and Thalassiosira pseudonana. Chemosphere, 14, 383-392.

WONG P.T.S., CHAN Y.K., KRAMAR O., BENGERT G.A., 1982. Structure-toxicity relationship of tin compounds on algae. Can. J. Fish. aquat. Sci., 39, 483-488.

ZUOLIAN C., JENSEN A., 1989. Accumulation of organic and inorganic tin in blue mussel, Mytilus edulis, under natural conditions. Mar. Pollut. Bull., 20, 281-286. 